\title{
Die KMU-Forschung - gestern und heute
}

Die Digitalisierung von KMU wird nicht erst seit ein paar Jahren, sondern schon lange untersucht. Es ist also kein neues Thema. Eine der ersten Studien zu KMU ist von Montazemi (1987). Er untersuchte die Anwendung des sogenannten Personal Computers (PC). Es folgten viele weitere Studien wie die von Raymond (1986) mit Fokus auf die Anwendung von Textverarbeitungsprogrammen wie Word in KMU. Ab 1994 rückte die Globalisierung von KMU in den Fokus der Forschung, da speziell Nischenexpertise oft weltweit skaliert werden musste. Durch eine globale Vernetzung wurden nach 2011 speziell die Potenziale der Innovation durch den Zusammenschluss von KMU untersucht. Seit 2013 finden sich vermehrt Studien zur Anwendbarkeit von verschiedensten Technologien (SAP, Sharepoint etc.) in KMU. Seit 2016 wird vermehrt der digitale Wandel von Arbeit, Führung und Organisation sowie Automatisierung diskutiert. Natürlich finden sich auch viele Randthemen, allerdings lag mein Fokus auf den Kernthemen.

\section{KMU-Forschung auf einen Blick}

- 1980-1993: Anwendung von PCs in KMU

- 1994-2010: Globalisierung von KMU

- 2011-2012: Innovation in KMU

- 2013-2015: IT in KMU

- 2016-2018: Digitalisierung, Führung, Arbeit und Organisation in KMU 